\title{
Fatigue Life Properties of Stainless Steels in Wide Ranged Biaxial Stress States using a Hollow Cylinder Specimen
}

\author{
Shunsuke Saito ${ }^{1}$, Fumio Ogawa ${ }^{2}$, Takamoto Itoh $^{2,2}$ \\ ${ }^{1}$ Graduate School of Science \& Engineering, Ritsumeikan University, Japan. \\ ${ }^{2}$ College of Science and Engineering, Ritsumeikan University, Japan
}

\begin{abstract}
Stress controlled multiaxial fatigue tests were carried out using hollow cylinder specimens of type 430 and 316 stainless steels at room temperature. A newly developed fatigue testing machine which can apply push-pull loading and inner pressure to the specimen was used. For inner pressure, oil was put inside of the specimen. 7 types of cyclic loading paths were employed by combining axial and hoop stresses; a Pull, an Innerpressure, a Push-pull, an Equi-biaxial, a Square-shape, a $\mathrm{L}_{\mathrm{T}}$-shape and a $\mathrm{LC}_{\mathrm{C}}$ shape. Fatigue lives vary depending on the loading path when those were evaluated by the maximum Mises' equivalent stress range on inner surface of the specimen. The fatigue lives of both the steels showed a similar tendency. However, the following difference was identified. Specifically, differences in fatigue lives of type 430 stainless steel between the uniaxial loading and the multiaxial tests were large, while those of type 316 stainless steel were small. To discuss difference in fatigue life properties between both steels, this study investigates the effect of the shear stress range, mean stress and additional hardening and which leads to evaluate the lives suitably.
\end{abstract}

\section{Introduction}

Machines and structures in service conditions undergo multiaxial fatigue damage. For example, many metal pipes are used in power plant and chemical plant. Those pipes receive multiaxial loading by movement of internal fluid [1]. However, most of the fatigue data which are used for those strength designs are obtained by uniaxial loading tests, and there is limited data under multiaxial loading [2-3]. The equivalent stress based on von Mises is generally used in the design for structures under multiaxial loading. In recent literatures of multiaxial fatigue, it is reported that fatigue life evaluated by the Mises' equivalent stress is overestimated [2-7] and the degree depends on loading path [6]. In the multiaxial fatigue under non-proportional loading in which directions of principal stress and strain are changed in a cycle, it has been reported that fatigue lives are reduced accompanying additional hardening which depends on both loading path and material. [4-8]. However, multiaxial principal stress ratio range which can be achieved by practical testing machine is limited. Therefore, there is need to develop testing machine for wider multiaxial principal stress ratio range.

\footnotetext{
$\overline{2 \text { Corresponding author: itohtaka@fc.ritsumei.ac.jp }}$
} 
The authors developed a special testing machine to resolve the above problem. The testing machine is an electric servo controlled multiaxial fatigue machine, which can combine the push-pull, the cyclic inner pressure by using oil and the reversed torsion loads. In this study, load controlled fatigue tests at room temperature were carried out using a hollow cylinder specimen under 7 types of loading path, which are a combination of the axial stress by pushpull loading and the hoop stress by inner pressure. The fatigue lives varied depending on the loading path when they were evaluated with equivalent stress ranges. The effect of loading path on fatigue life is discussed based on the experimental results.

\section{Multiaxial fatigue testing machine}

Fig. 1 and Fig. 2 show a schematic view and a photo of the multiaxial fatigue testing machine for the push-pull and the reversed torsion tests with the inner pressure. The testing machine is equipped with three actuators. The upper part of testing machine possesses the actuator of inner pressure and the two actuators in the lower side are used to apply the push-pull and the reversed torsion loading to the hollow cylinder specimen. A pressure gauge and a load cell are equipped to measure inner pressure, axial load and torque. Those measured data is used for evaluation of fatigue life and feedback to control the test by using inner pressure and axial load. Fig. 3 show a schematic view of the extensometer for measurement of the axial displacement. This extensometer is attached directly onto the specimen as shown in Fig. 3 and a gauge length is $7 \mathrm{~mm}$. Fig. 4 show a schematic view of the extensometer for measurement of the hoop displacement. The extensometer clamps the specimen by using a rubber band on the extremity of the device as shown in Fig. 4.

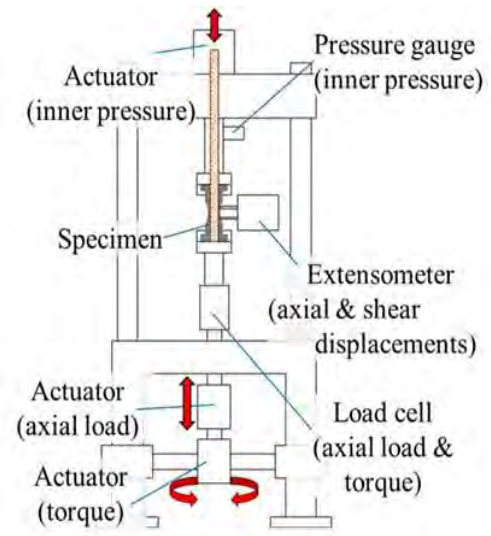

Fig. 1 Schematic view of multiaxial fatigue testing machine.

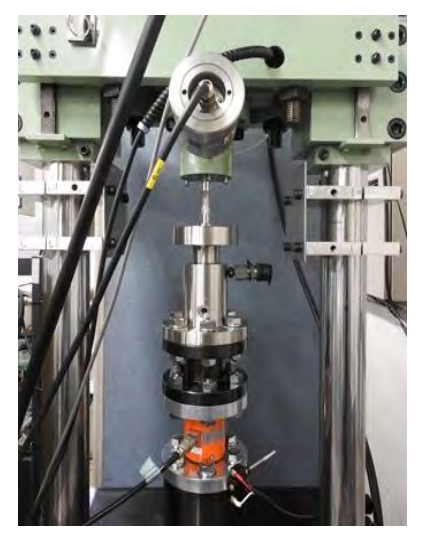

Fig. 2 Photo picture of multiaxial fatigue testing machine.

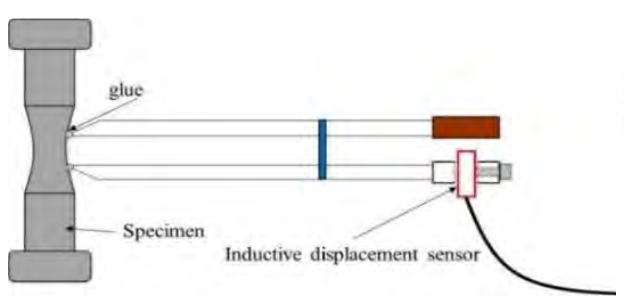

Fig. 3 Schematic view of extensometer to measure axial displacement.

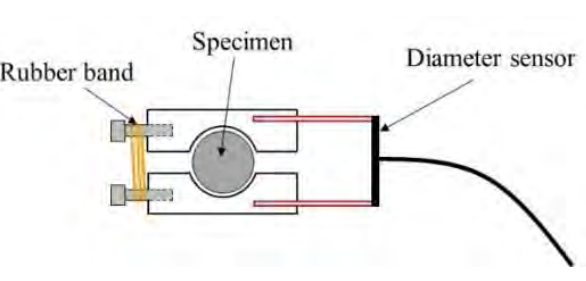

Fig. 4 Schematic view of extensometer to measure hoop displacement. 


\section{Experiments}

\subsection{Specimen}

The material tested was a type 430 stainless and a type 316 stainless steels, $430 \mathrm{SS}$ and 316SS. Fig. 5 and Fig. 6 show a schematic view and a photo of the shape and dimensions of the hollow cylinder specimen which has a $12 \mathrm{~mm}$ inner diameter, a $14 \mathrm{~mm}$ outer diameter and an $8.5 \mathrm{~mm}$ gauge part. The parallel gauge part was polished up to $1 \mu \mathrm{m}$ alumina particles. Inner surface of the specimen was polished by emery paper from \#600 to \#2000.

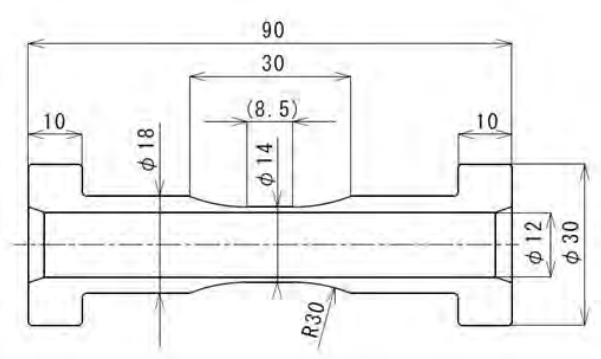

Fig. 5 Shape and dimensions of specimen (mm).

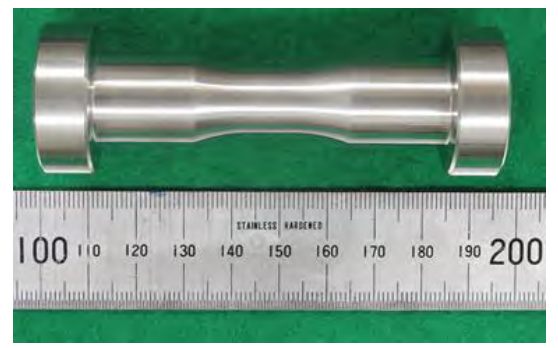

Fig. 6 Photo picture of specimen.

\subsection{Multiaxial stress}

In this study, the axial stress $\sigma_{\mathrm{z}}$, the hoop stress $\sigma_{\theta}$ and the radius stress $\sigma_{\mathrm{r}}$ are defined by the following equations,

$$
\begin{gathered}
\sigma_{z}=\frac{F}{\pi\left(r_{\mathrm{o}}^{2}-r_{\mathrm{i}}^{2}\right)}+\frac{P r_{\mathrm{i}}^{2}}{r_{\mathrm{o}}^{2}-r_{\mathrm{i}}^{2}} \\
\sigma_{\theta}=\frac{P r_{\mathrm{i}}^{2}}{r_{\mathrm{o}}^{2}-r_{\mathrm{i}}^{2}}\left(1+\frac{r_{\mathrm{o}}^{2}}{r^{2}}\right) \\
\sigma_{r}=\frac{P r_{\mathrm{i}}^{2}}{r_{\mathrm{o}}^{2}-r_{\mathrm{i}}^{2}}\left(1-\frac{r_{\mathrm{o}}^{2}}{r^{2}}\right)
\end{gathered}
$$

where $F$ and $P$ are the axial load and the inner pressure, respectively. $r$ is an arbitrary radius in the specimen thickness direction, $r_{\mathrm{i}}$ and $r_{\mathrm{o}}$ are radii at the inner and the outer surfaces of the specimen, respectively $\left(r_{\mathrm{i}}=6 \mathrm{~mm}\right.$ and $\left.r_{\mathrm{o}}=7 \mathrm{~mm}\right)$. Therefore, the maximum Mises' equivalent stress is varied from inner to outer surfaces, which is defined as,

$$
\sigma_{\text {eqmax }}^{i}=\operatorname{Max}\left[\frac{1}{2}\left\{\left(\sigma_{z}-\sigma_{\theta}\right)^{2}+\left(\sigma_{\theta}-\sigma_{\mathrm{r}}\right)^{2}+\left(\sigma_{\mathrm{r}}-\sigma_{\mathrm{z}}\right)^{2}\right\}\right]^{\frac{1}{2}}
$$

where $i$ indicates the position on the specimen (I: inner surface, M: middle between inner and outer surfaces, O: outer surface).

\subsection{Experimental procedure}

Load- and pressure-controlled fatigue tests were carried out at room temperature. Fig. 7 shows 7 types of loading waveforms and loading paths employed: a Pull, an Inner-pressure, a Push-pull, an Equi-biaxial, a Square-shape, a $\mathrm{L}_{\mathrm{T}}$-shape and a $\mathrm{L}_{\mathrm{C}}$-shape. The Pull is a cyclic pull loading in which only axial stress $\sigma_{z}$ is applied. The Inner-pressure is a cyclic inner 
pressure test in which only hoop stress $\sigma_{\theta}$ is applied. The Push-pull is a cyclic push-pull loading. These three loadings are classified as the uniaxial loading. The Equi-biaxial, the Square-shape, the $\mathrm{L}_{\mathrm{T}}$-shape and the $\mathrm{L}_{\mathrm{C}}$-shape are multiaxial loading tests in which cyclic pull loading and inner pressure are combined. In the Equi-biaxial test, axial stress $\sigma_{\mathrm{z}}$ and hoop stress $\sigma_{\theta}$ are applied at the same time. In the Square-shape test, axial stress $\sigma_{z}$ and hoop stress

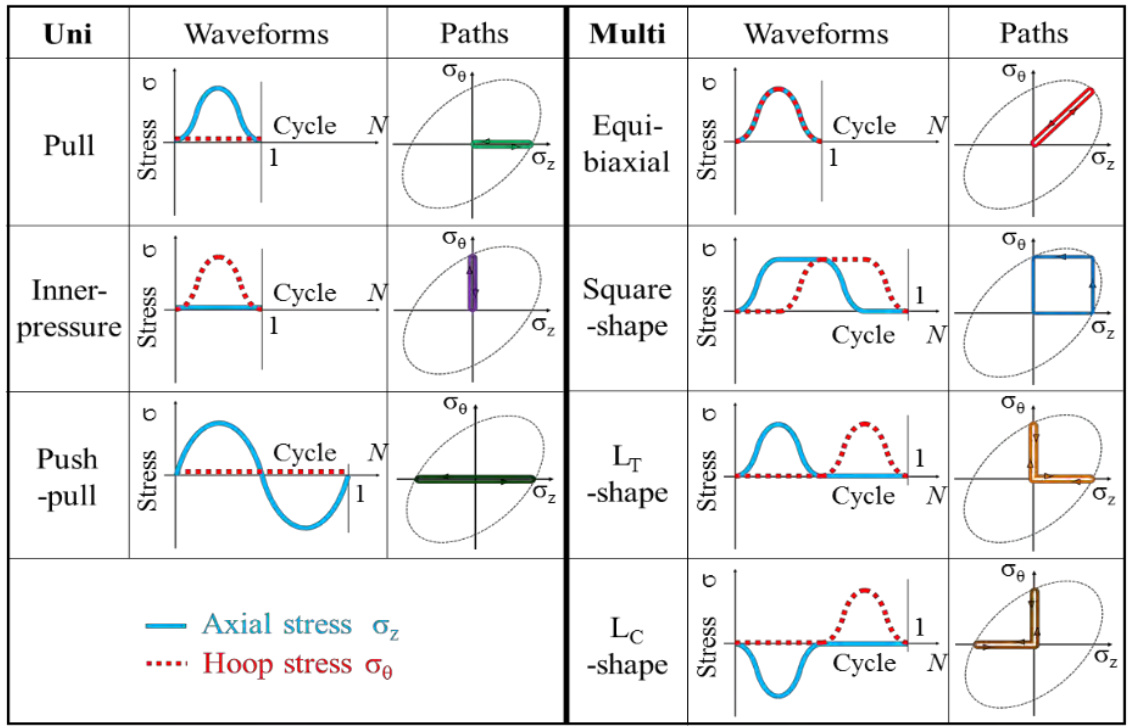

Fig. 7 Waveforms and paths for 7 loadings.

$\sigma_{\theta}$ are applied with 90-degree phase difference so that the shape of loading path becomes square. In the $\mathrm{L}_{\mathrm{T}}$-shape test, axial stress $\sigma_{\mathrm{z}}$ and hoop stress $\sigma_{\theta}$ are applied alternately so that the shape of loading path becomes the shape of the letter " $L$ ". In the $\mathrm{L}_{C}$-shape test, axial stress $\sigma_{\mathrm{z}}$ by compression loading and hoop stress $\sigma_{\theta}$ are alternately applied. In the Equi-biaxial, the Square-shape, the $\mathrm{L}_{\mathrm{T}}$-shape and the $\mathrm{L}_{\mathrm{C}}$-shape tests, the axial stress and the hoop stress amplitudes are set to be the same as that on the outer surface. In the test condition except for the pull test, the maximum Mises' equivalent stress at inside of the specimen $\sigma_{\text {eq } \max }^{\mathrm{I}}$ is 1.3 times larger than that at the outside of the specimen $\sigma^{\mathrm{O}}$ eq max.

One cycle is counted when a block of loading path is finished. Fatigue life, $N_{\mathrm{f}}$, was defined as the cycle at which the maximum inner pressure drops to $80 \%$ or less of the set value by the oil leakage due to a formation of through crack or rupture of the specimen. In the Pull test, constant inner pressure of $1 \mathrm{MPa}$ was applied during test to unify definition of the fatigue life.

\section{Test result}

\subsection{Fatigue life}

To evaluate the fatigue life, the Mises' equivalent stress range on the inner surface $\Delta \sigma_{\text {eq }}^{\mathrm{I}}$ was used because the equivalent stress takes the largest along specimen thickness direction. In this study, $\Delta \sigma_{\text {eq }}^{\mathrm{I}}$ corresponds with the maximum Mises' equivalent stress $\sigma_{\text {eqmax }}^{\mathrm{I}}$ in all the paths except for the Pull and the Push-pull test. Fig. 8 shows a relationship between $\Delta \sigma_{\text {eq }}^{\mathrm{I}}$ and fatigue life $N_{\mathrm{f}}$ of 430SS for loading paths; the Pull, the Inner-pressure, the Equi-biaxial, the Square-shape and the $\mathrm{L}_{\mathrm{T}}$-shape test. $N_{\mathrm{f}} \mathrm{S}$ in the Pull, the Inner-pressure and the Equi-biaxial tests are correlated on a unique line. However, although $N_{\mathrm{f}} \mathrm{S}$ in the Square-shape and $\mathrm{L}_{\mathrm{T}}$-shape 
tests are also correlated with a unique line, the lives are much shorter than those in former three loading paths; they have almost double-digit decrease in fatigue lives. The correlation shows that the fatigue life is affected largely by the loading path under the wide ranged biaxial stress state tested.

Fig. 9 shows a relationship $\Delta \sigma_{\text {eq }}^{\mathrm{I}}$ and $N_{\mathrm{f}}$ of $316 \mathrm{SS}$ for the Pull, the Inner-pressure, the Pushpull, the Equi-biaxial, the Square-shape, the $\mathrm{L}_{\mathrm{T}}$-shape and the $\mathrm{L}_{\mathrm{C}}$-shape tests. The correlation for $316 \mathrm{SS}$ also shows the same tendency as that for 430SS under the same loading paths, but the reduction in $N_{\mathrm{f}}$ in the Square-shape and the $\mathrm{L}_{\mathrm{T}}$-shape tests is much less: one-digit decrease. Moreover, the fatigue life in the Pull test tend to be underestimated, which will be discussed later related to crack behavior. The $N_{\mathrm{f}} \mathrm{S}$ in the $\mathrm{L}_{\mathrm{C}}$-shape, the Inner-pressure and the Equibiaxial test are correlated on a unique line.

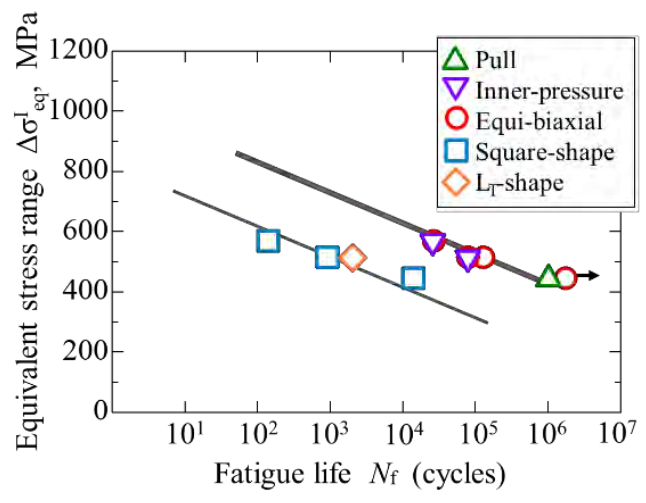

Fig. 8 Relationships between equivalent stress range and fatigue life of 430SS.

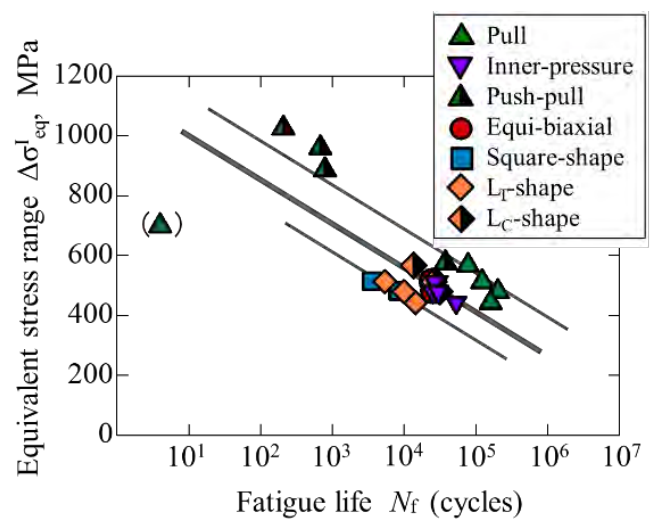

Fig. 9 Relationships between equivalent stress range and fatigue life at of $316 \mathrm{SS}$.

\subsection{Shear stress and mean stresses}

Fig. 10 shows three shear stress range; $\tau_{\mathrm{z} \theta}, \tau_{\theta \mathrm{r}}, \tau_{\mathrm{rz}}$ of each loading path. The shear stress range $\tau_{z \theta}$ of the Square-shape and the $\mathrm{L}_{\mathrm{T}}$-shape test are twice compared with those of the Pull, the Inner-pressure, the Equi-biaxial and the $\mathrm{L}_{\mathrm{C}}$-shape test; the $N_{\mathrm{f}} \mathrm{S}$ of the Square-shape and the $\mathrm{L}_{\mathrm{T}}$-shape resulted in shorter, which indicates that life evaluation needs to consider the direction in shear stress. Fig. 11 (a) and (b) replot the $N_{\mathrm{f}}$ in Figs. 8 and 9 using an equivalent stress range, $\Delta \sigma_{\text {eq }}^{\mathrm{I}}{ }^{*}$ defined by introducing the direction change in shear stress. This idea is corresponded with I-S method was proposed by Itoh, Sakane et. al [9]. In contrast to the correlations in Figs. 8 and 9, the fatigue lives in the Square-shape and the $\mathrm{L}_{\mathrm{T}}$-shape test are overestimated obviously. In the Square-shape and the $\mathrm{L}_{\mathrm{T}}$-shape test shown in Fig. 10, stress waveforms applied on $\tau_{\mathrm{z} \theta}, \tau_{\theta \mathrm{r}}$ and $\tau_{\mathrm{rz}}$-plane has difference phase despite directions of principal stresses are fixed. The $\mathrm{L}_{\mathrm{C}}$-shape test also has the phase difference but $\Delta \sigma_{\mathrm{eq}}^{\mathrm{I}}{ }^{*}$ is a half of those of above two paths resulting in elastic deformation dominant. Furthermore, the number of cycle-counting in the $\mathrm{L}_{\mathrm{c}}$-shape test becomes twice. This suggests that the considering of the change in maximum shear stress plane also becomes a key issue to evaluate fatigue life under wide ranged biaxial stress state.

In Fig. 11, $N_{\mathrm{f}} \mathrm{s}$ in the Push-pull, the Square-shape and the $\mathrm{L}_{\mathrm{T}}$-shape test are longer than that in the other tests, which may be resulted from zero mean stress in $\tau_{z \theta}$, whereas the other tests have mean stress. The authors consider the effect of mean stress. Figs. 12 (a) and (b) show fatigue limit diagrams for 430SS and 316SS. For 430SS, fatigue limit $\left(\sigma_{\mathrm{w} 0}\right)$ decreases from $263 \mathrm{MPa}$ to $180 \mathrm{MPa}$ when mean stress $\sigma_{\mathrm{m}}$ is $131.5 \mathrm{MPa}$. Therefore the fatigue limit in the Square-shape and the $\mathrm{L}_{\mathrm{T}}$-shape test are decreased by $83 \mathrm{MPa}$ resulting in newly indicated 


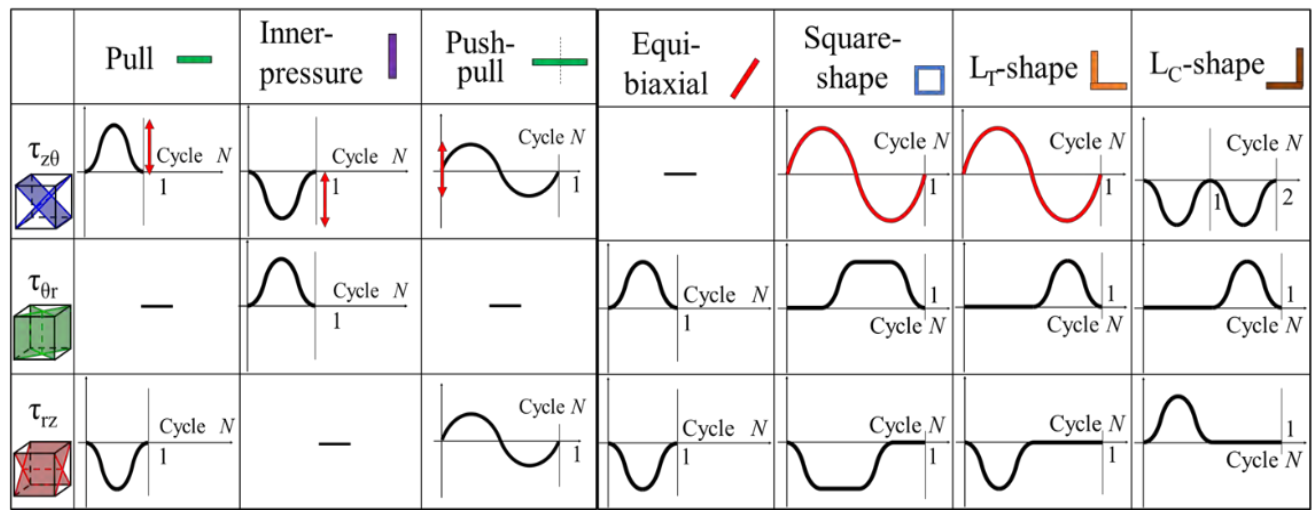

Fig. 10 The range of maximum shear stress.

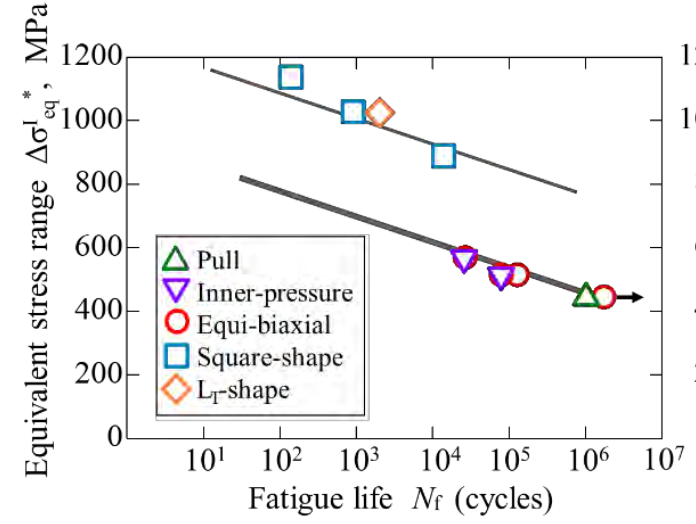

(a) 430SS

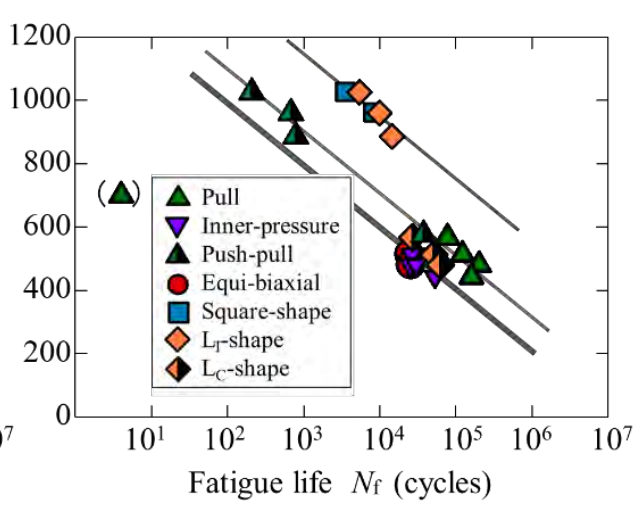

(b) $316 \mathrm{SS}$

Fig. 11 Relationships between equivalent stress range taking account of shear stress direction and fatigue life.

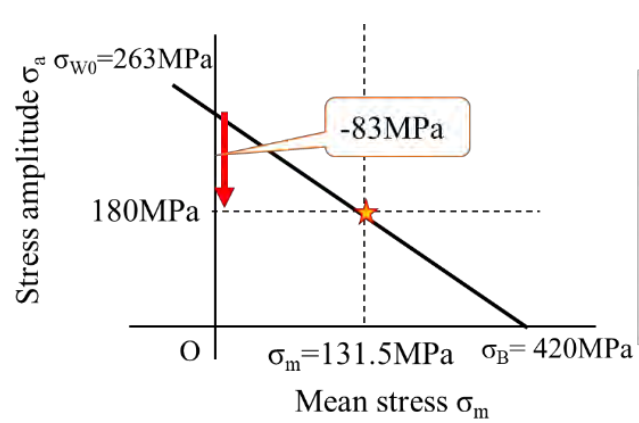

(a) $430 \mathrm{SS}$

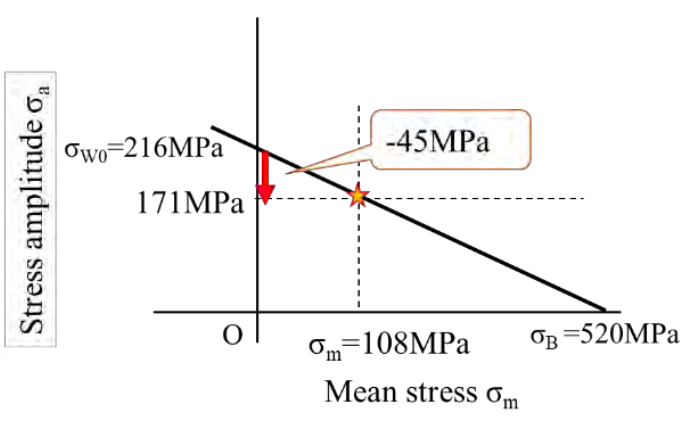

(b) $316 \mathrm{SS}$

Fig. 12 Relationships between stress amplitude and mean stress.

stress range $\Delta \sigma_{\text {eq }}^{\mathrm{I}}{ }^{* *}=166 \mathrm{MPa}$ by take into account of the mean stress. In the similar way, $\Delta \sigma_{\text {eq }}^{\mathrm{I}}{ }^{* *}=90 \mathrm{MPa}$ for $316 \mathrm{SS}$.

Figs. 13 (a) and (b) replot the $N_{\mathrm{fs}}$ in Figs. 11 (a) and (b) using the equivalent stress range, $\Delta \sigma_{\text {eq }}^{\mathrm{I}}{ }^{* *}$. In Fig. 13 (a), fatigue lives are almost correlated by a unique line, which indicates 


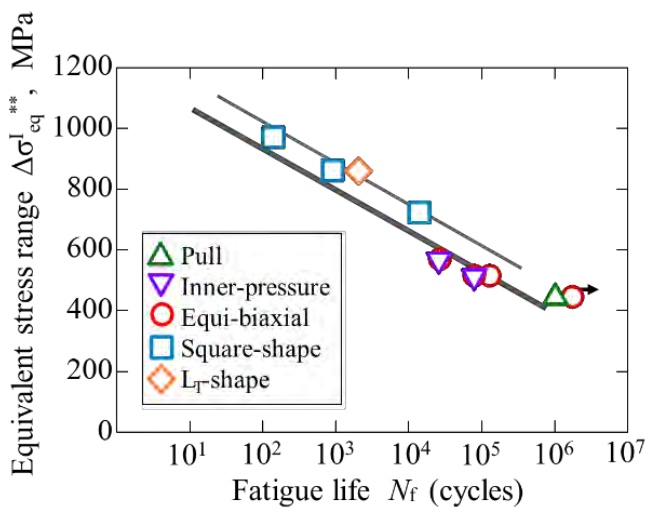

(a) 430SS

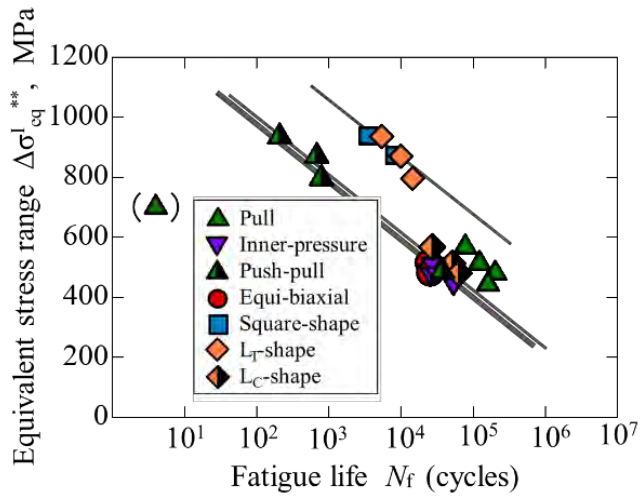

(b) $316 \mathrm{SS}$

Fig. 13 Relationships between equivalent stress range taking account of mean stress and fatigue life.

the overestimation in the Square-shape and the $\mathrm{L}_{\mathrm{T}}$-shape test are solved. On the other hand, correlations for 316 SS in Fig. 13 (b), the fatigue lives in the Push-pull, Inner-pressure and the Equi-biaxial tests are correlated by a unique line, but fatigue lives in the Square-shape and the $\mathrm{L}_{\mathrm{T}}$-shape test are still overestimated. This overestimation may be caused by an additional hardening due to direction change in the maximum principal stress and shear stresses in a cycle. Fig. 14 shows photo picture of specimen surfaces in the Square-shape test for 430SS and 316SS. Specimen surface for 430SS shows a drastic bulging at the gauge part which may be caused by cyclic inelastic deformation. In contrast, the bulging for 316SS is much less because the cyclic hardening occurs easily for austenitic stainless steel [10].

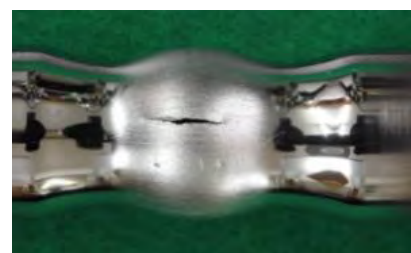

(a) 430SS

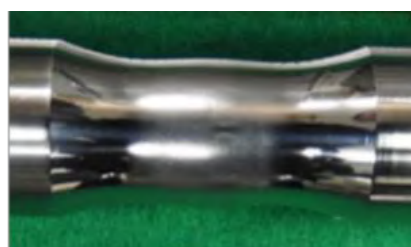

(b) $316 \mathrm{SS}$

Fig. 14 Photo picture of supecimen surface after fatigued (512.7MPa). 
The above suggests that the effect of additional hardening also should be taken into account for life evaluation as well as that under non-proportional loading, even though principal directions of stress and strain are fixed in which only the directions of maximum principal stress and strain are changed. To discuss this effect, more detail discussion as well as caring out additional experimental tests are needed.

\section{Conclusions}

1. Fatigue life depends on the loading path and that was short in order of the Square-shape test, the $\mathrm{L}_{\mathrm{T}}$-shape test, the Inner pressure, the Equi-biaxial, $\mathrm{L}_{\mathrm{C}}$-shape, Push-pull and the Pull tests.

2. In order to evaluate accurately the fatigue life with the Mises' equivalent stress range referred to the inner surface, the effect of the directional change of the maximum principal stress and shear stress (I-S method) as well as the mean stress must be considered.

3. The cause of the higher fatigue lives in case of Square-shape and $\mathrm{L}_{\mathrm{T}}$-shape tests compared to Push-pull tests in $316 \mathrm{SS}$ could be the additional cyclic hardening due to the change in direction of the maximum principal stress.

\section{References}

1. T. Itoh, Development of internal and external pressure and axial and torsional composite multiaxial fatigue test equipment, Fukui University Jpn. (2011)

2. A. Nitta, T. Ogata, K. Kuwabara, The effect of axial-torsional straining phase on elevated-temperature biaxial low-cycle fatigue life in SUS 304 stainless steel, J. Soc. Mater. Sci. 36: 376-382 (1989).

3. C.H. Wang, M.W. Brown, A path-independent parameter for fatigue under proportional and non-proportional loading, Fatigue \& Fract. Eng. Mater. \& Struct. 16: 1285-1298 (1993).

4. T. Itoh, T. Nakata, M. Sakane, M.Ohnami, Nonproportional low cycle fatigue of 6061 aluminum alloy under 14 strain paths, Euro. Struct. Integrity Soc. 25: $41-54$ (1999).

5. N. Shamsaei, M. Gladskyi, K. Panasovskyi, S. Shukaev, A.Fatemi, Multiaxial fatigue of titanium including step loading and load path alteration and sequence effects, Int. J. Fatigue 32: 1862-1874 (2010)..

6. A. Karolczuk, Analysis of revised fatigue life calculation algorithm under proportional and non-proportional loading with constant amplitude, Int. J. Fatigue 88: 111-120 (2016).

7. X. Chen, Q. Gao, X. Sun, Low-cycle fatigue under non-proportional loading, Fatigue \& Fract. Eng. Mater. \& Struct. 19: 839-854 (1996).

8. A. Fatemi, D.F. Socie, A critical plane approach to multiaxial fatigue damage including out-of-phase loading, Fatigue \& Fract. Eng. Mater. \& Struct. 11: 149-165 (1988).

9. T. Itoh, M. Sakane, Y. Shimizu, Definition of stress and strain ranges for multiaxial fatigue life evaluation under non-proportional loading, J. Soc. Mater. Sci. 62: 117-123 (2013).

10. M. Sakane, Effect of Multiaxial Stress on Low Cycle Fatigue Life and Creep Rupture Lifetime, J. Soc. Mater. Sci. 66: 70-79 (2017) 